\title{
Lectura del paisaje urbano de la colonia Americana
}

Yolanda Torres López

Universidad de Guadalajara

\section{Resumen}

Así como un libro está compuesto de un conjunto de palabras y oraciones que intentan decir algo con sentido, las edificaciones son textos compuestos de un lenguaje arquitectónico cuyo significado va más allá de la imagen, pues involucran un uso y una disposición.

El propósito de esta discusión es hacer una reconstrucción semiótica del barrio como un conjunto de obras arquitectónicas que conforman un paisaje urbano, ya sean espacios de integración o espacios de tránsito. El presente estudio se realiza desde la antropología cultural, por lo que es importante aclarar que no parte exclusivamente del urbanismo y la arquitectura.

El paisaje urbano está integrado por edificaciones, manzanas, calles, avenidas, parques y plazas. Este conjunto edilicio, en compañía de las representaciones mentales y estructuradas que los usuarios de la ciudad tienen, hacen esta legibilidad de los lugares y conforman un texto. La selección del corpus de estudio en este caso no está determinada por las divisiones administrativas de los barrios o colonias, sino que toma como referentes hitos, espacios y funciones de la ciudad que marcan límites y lugares de destacada significación cultural, ya sea por su tradición, valor patrimonial o porque constituyen espacios de prácticas sociales significativas.

Palabras clave: Arquitectura, patrimonio, paisaje urbano, semiótica, identidad. 


\section{Abstract}

Just like a book is composed by a set of words and sentences that try to say something that makes sense, buildings are compound texts of an architectural language whose meaning goes beyond the image, since they involve a use and a disposition.

The purpose of this discussion is to make a semiotic reconstruction of the neighborhood as a set of architectural works that make up an urban landscape, whether they are spaces of integration or spaces of transit. The present study is done from the cultural anthropology, that's why it's important to clarify that the study doesn't take off exclusively from the urbanism and the architecture.

The urban landscape it's formed by edifications, blocks, streets, avenues, parks, and squares. This group of buildings, along with the mental structured representations that the users of the city have, makes this readability of the places, and conforms a text. The selection of the study corpus in this case it's not determinate by the administrative divisions of the neighborhoods or colonies, but instead it takes as referents milestones, spaces and functions of the city that mark limits and places of outstanding culture significance, either because of its tradition or patrimonial value or because they constitute spaces of significative social practice

Keywords: Architecture, heritage, urban landscape, semiotics, identity.

\section{Introducción}

Aunque se han realizado estudios semióticos aplicados a la arquitectura ${ }^{1}$ y dentro del estructuralismo se hicieron aportaciones importantes al análisis de la arquitectura y la planificación urbana, ${ }^{2}$ en este trabajo se busca aplicar algunos conceptos planteados por Tzvetan Tororov al estudio del espacio urbano, mediante algunas propuestas transdisciplinarias de lo que podríamos considerar una poética del espacio.

Tzvetan Todorov señala que el término poética se entendió como sinónimo de literatura en general, no solo la versificada, sino también la escrita en prosa, asegurando además que la ciencia puede utilizar el texto como materia de análisis, partiendo del hecho de que toda obra de arte sirve para ilustrar, «participa la poética del proyecto semiótico general, que unifica todas las investigaciones cuyo punto de partida es el signo». ${ }^{3}$

Charles Sanders Peirce afirma en su tratado La ciencia de la semiótica (1986) que un signo es algo que para alguien representa o se re-

1 Eco, La estructura ausente. Introducción a la semiótica; Broadbent, Bunt, y Jencks, El lenguaje de la arquitectura: un análisis semiótico.

2 Aldo van Eyck, Alison y Peter Smithson, miembros del Grupo Team 10.

3 Todorov, Poética estructuralista, 42. 
fiere a algo en algún aspecto o carácter. El signo está en lugar de algo, su objeto, no en todos los aspectos, sino solo con referencia a una suerte de idea.

Así como la semiótica estudia la correlación entre significante y significado, la literatura utiliza el lenguaje, que es un sistema simbólico secundario para hacer representaciones a través de textos. Para Todorov, existen tres aspectos del texto literario a analizar: el aspecto sintáctico, el aspecto verbal y el aspecto semántico.

a. La sintáctica en la literatura estudia las estructuras y formas de organización en los textos o sistemas cerrados, el orden lógico y temporal, el orden espacial, la sintaxis narrativa, especificaciones y reacciones.

b. En el aspecto verbal se estudia el estilo o modalidad de cada narración, es decir, la forma. Se analizan el modo, el tiempo, las visiones y la voz.

c. El aspecto semántico estudia los significados en los sistemas abiertos. Su materia de análisis son los registros del habla. Sus categorías son: el discurso, la presencia de figuras retóricas, la presencia o ausencia de referencias que crean un discurso polivalente y la subjetividad del lenguaje.

Desde esta lógica, la arquitectura también está compuesta de un lenguaje secundario y es susceptible de una poética. Los elementos físicos que delimitan un espacio, el tipo y el material utilizado en cimientos, muros, techos o pisos son signos que exigen un análisis que refleje una cultura, una ubicación geográfica y una dinámica social. Decía el poeta, dramaturgo y novelista francés Víctor Hugo que «La arquitectura es el gran libro de la humanidad», porque es posible leer a través de esta a la sociedad que la produjo.

La percepción de los usuarios inmersos en la poética del espacio urbano de la colonia Americana de Guadalajara, será materia de análisis a partir de las aproximaciones estructuralistas de Todorov, entendiendo a las edificaciones del conjunto urbano como un sistema de signos delimitado.

\section{Espacio urbano como texto}

El paisaje urbano está integrado por edificaciones, manzanas, calles, avenidas, parques y plazas. Este stock edilicio conforma un texto. Javier Bolaños Palacio afirma lo siguiente:

Se podría decir entonces que entender los hechos edilicios en la historia, es entender el desarrollo de los procesos constructivos ligados a la técnica, la ideología, la política y el contexto 
económico de las sociedades que los han producido en un momento y lugar determinado. ${ }^{4}$

En 1960 Kevin Lynch desarrolló una teoría acerca de los elementos tangibles de la ciudad que estructuran las representaciones mentales de sus usuarios, es decir, la legibilidad de los lugares. Para entender este trabajo se hace necesaria la comprensión de los diferentes elementos que estructuran la percepción física de los elementos del paisaje urbano. Lynch identifica cinco tipologías de percepción de elementos estructurales del espacio urbano: hitos (mojones), carreteras (sendas), límites (bordes), barrios y nodos. El análisis propuesto por Lynch es de carácter cualitativo.

Los límites o bordes son los elementos lineales que el observador no usa o considera sendas. Son los límites entre dos fases, rupturas lineales de la continuidad, como cruces de calles, muros o vallas, pueden ser más o menos penetrables y separan una región de otra, o bien pueden ser suturas, líneas según las cuales se relacionan y unen dos regiones. Estos elementos fronterizos, si bien posiblemente no son tan dominantes como las sendas, constituyen importantes rasgos organizadores, en especial en la función de mantener juntas zonas generalizadas, como el contorno de una ciudad trazados por el agua o una muralla. ${ }^{5}$

La delimitación de este conjunto de edificaciones constituye el campo semántico acotado del presente estudio, y es la siguiente: al norte por la calle Morelos, al este por la avenida Federalismo, al sur por la avenida de la Paz y al este por la avenida Enrique Díaz de León. El conjunto de signos de este espacio urbano son las edificaciones que lo conforman, pero también sus sendas, que en este caso no son carreteras sino calles (arroyos y aceras), sus hitos barriales (como el Templo Expiatorio, el parque de la Revolución, el edificio de la Rectoría de la Universidad de Guadalajara, el edificio administrativo de la Universidad de Guadalajara, etcétera) y su nodos, que en este caso corresponden a lugares de tránsito tanto peatonal como vehicular en el que se cruzan distintas sendas. (véase la ilustración 1).

Dentro de esta concepción se debe considerar que existen espacios de integración e integrados, que son preferentemente lugares que tienen una significación tradicional en una comunidad, ya sea por cuestiones de costumbres, de prácticas sociales, culturales o de convivencia.

La colonia Americana es una zona que se caracteriza por la heterogeneidad de sus actividades. La necesidad de acotar el área de estudio responde a la búsqueda de procesos de conformación y usos del espacio específicos. Comprender la conformación histórica de este

4 Bolaños Palacios, Pachajoa, y Aguilera Martínez, «Concepto de Edilicia», 3839.

5 Kevin Lynch, La imagen de la ciudad, 60. 


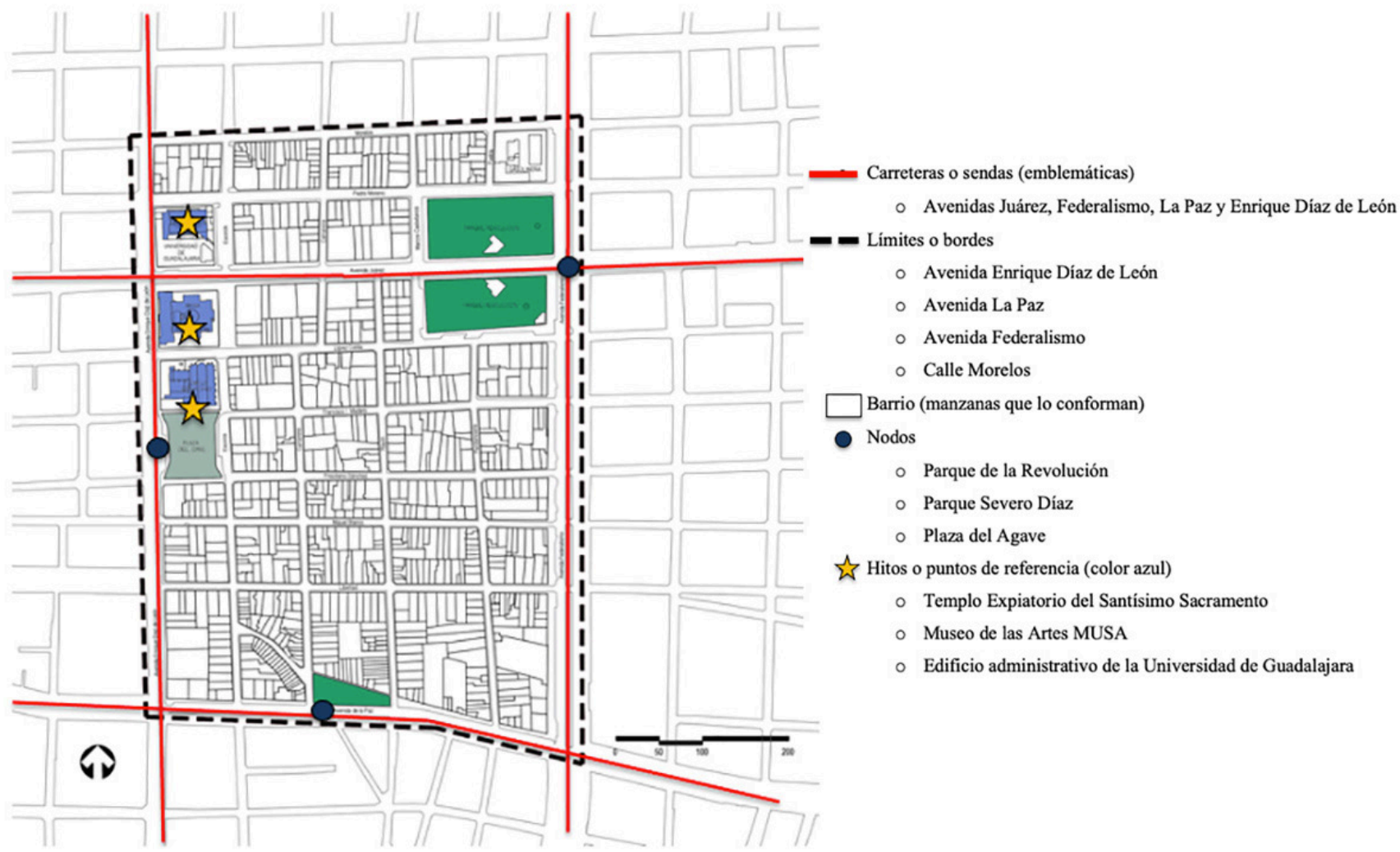

lustración 1. Identificación de elementos urbanos del área de estudio según Kevin Lynch (2015). Fuente: Elaboración propia. 
asentamiento fue el primer objetivo de la investigación para entender la variedad de espacios y procesos sociales.

La reconformación de la zona está directamente relacionada con el tipo de fisonomía del espacio, sus habitantes, sus ritmos y sus estilos de vida. La transformación de la colonia Americana no es independiente del crecimiento de la ciudad de Guadalajara, aunque en la actualidad responde a cierta tendencia de desarrollo propio. Sus principales edificios, iglesias y monumentos son símbolos de la identidad cultural y arquitectónica de la zona. La cercanía al edificio administrativo de la Universidad de Guadalajara y al Museo de las Artes la consolidó como un centro cultural importante de la zona centro de la ciudad, no solo porque allí viven personas vinculadas con el quehacer cultural o intelectual, sino porque es un espacio donde se desarrollan actividades culturales promovidas por el Estado, así como por asociaciones civiles y grupos de iniciativa privada.

Uno de los principales espacios de integración del área de estudio seleccionada es el que se encuentra en torno al Templo Expiatorio, la edificación religiosa más importante de la zona. Es un edificio de arquitectura ecléctica neogótica proyectado por el arquitecto italiano Adamo Boari, también autor del Palacio de Bellas Artes y del Palacio Postal en la Ciudad de México.

El área pública ubicada frente al recinto religioso es la plaza del Agave, proyecto del arquitecto Ignacio Díaz Morales, que corresponde al atrio del templo, y que actualmente desempeña un lugar de reunión y espacio de tránsito, no solamente porque tiene dos niveles de estacionamiento subterráneo, sino también porque en torno a él se ubican diversas fincas con funciones comerciales, educativas y de servicios que permiten y facilitan la integración de la comunidad, al igual que de visitantes externos. (Véase la ilustración 2).

En esa lógica, se identifica la plaza del Agave como un nodo, Lynch afirma:

Los nodos son puntos estratégicos de una ciudad a los que el observador puede ingresar y constituyen focos intensivos de los que se parte o a los que se llega. Son ante todo confluencias, sitios de una ruptura de transporte, un cruce o una convergencia de sendas, momentos de paso de una estructura a otra. ${ }^{6}$

Por otra parte, cuando hablamos de espacios integrados, estamos extrapolando un concepto que proviene de otras disciplinas de análisis cultural. En el ámbito de la literatura se habla de libros integrados cuando esas obras están conformadas por textos que tienen su propia independencia, pero que establecen relaciones significativas entre sí. Pueden haber sido reunidos por editores, autores o simplemente por antologistas. En este sentido, se está hablando de un espacio integrado, es decir, distintos tipos de textos edificatorios, algunos de producción popular y otros de ingeniería, así como obras de diseño arquitectónico. Resulta importante delimitar los elementos que se integran al contexto. En este caso, no se trata de la totalidad de las

6 Lynch, La imagen de la ciudad, 60. 


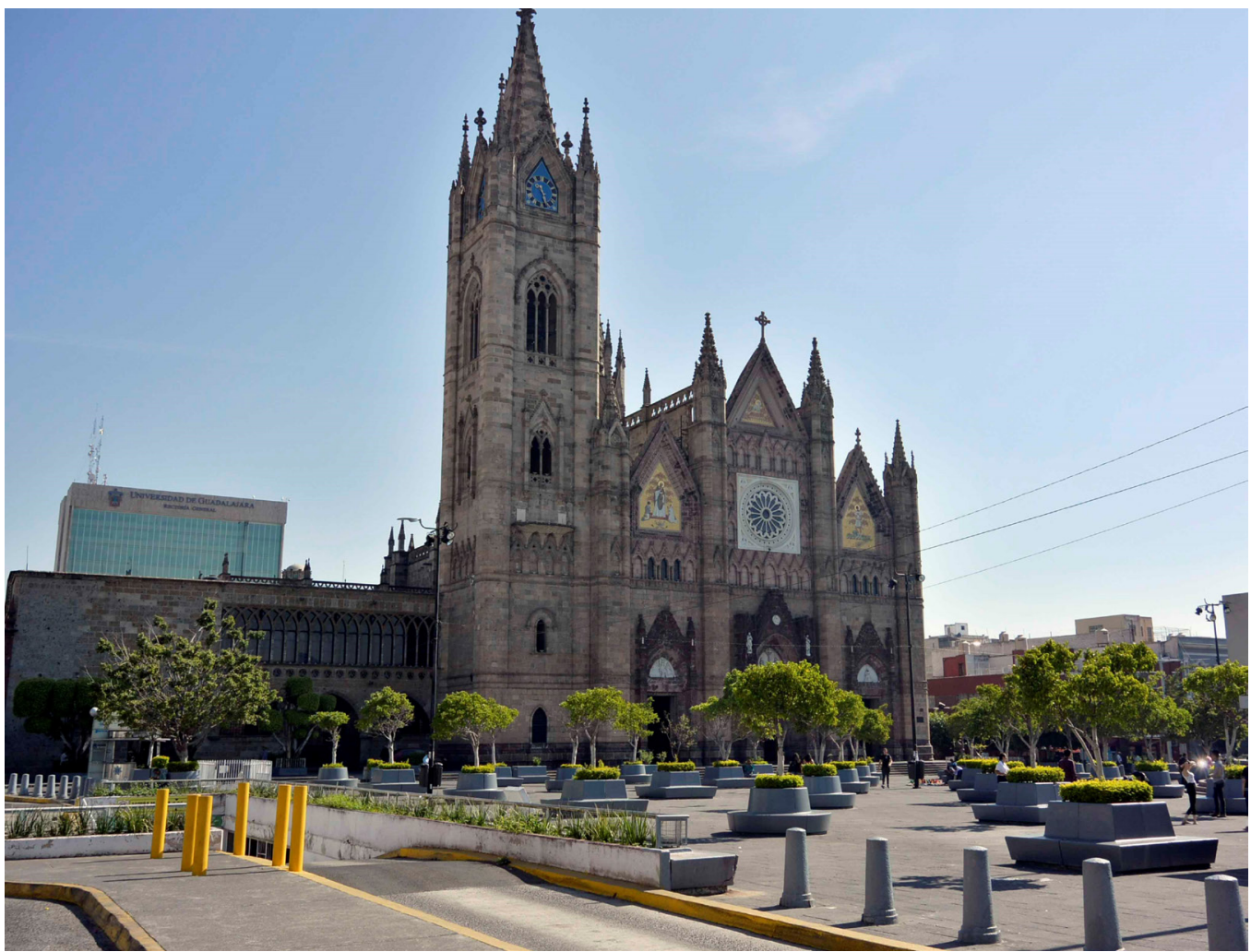

Ilustración 2. Templo Expiatorio y plaza del Agave. Fuente: propiedad de la autora. edificaciones, sino principalmente de las fachadas de estas edificaciones, y también se destacan las funciones que tienen esas edificaciones, algunas de ellas viviendas y otras comercios, que interactúan con el espacio público. Es importante destacar que tanto las fachadas como las funciones de las viviendas varían con el tiempo, por lo que aportan rasgos semánticos variables.

De esa manera, el objeto de estudio se adscribe a una lógica de estudio estructuralista, como la que propone Todorov al analizar la forma de relación que los componentes del texto establecen entre sí.

Aunque es posible identificar estilemas, que son signos indicadores de un estilo arquitectónico, ya sea modernista, regionalista, neogótico o art nouveau, es decir, movimientos artísticos con ciertos rasgos formales y temas, este estudio pretende principalmente conocer y analizar las prácticas sociales y culturales que se realizan en torno al espacio urbano. Estas prácticas varían según la hora y día de la semana e incluso en relación con ciertas festividades tanto de carácter religioso como popular o comercial, por lo que es necesario registrar estas variantes. Este estudio cultural exige considerar el desenvolvimiento diacrónico del espacio, dicho de otro modo, no se referirá únicamente a un momento determinado, sino a diferentes momentos de la vida cotidiana inscritos en este contexto urbano.

Según la clasificación de áreas del Plan Parcial de Desarrollo Urbano 2017 del Gobierno de Guadalajara, el área de estudio está inscrita en el Distrito Urbano 1 «Centro Metropolitano», dentro del Subdistrito Urbano 04 «Santa Teresita» y el Subdistrito Urbano 07 «La Moderna». (Véanse las ilustraciones 3 y 4 ).

Las áreas de Impacto Medio de Administración Pública (E3-AP), son zonas regionales que contienen equipamiento con un alcance que rebasa al propio centro de población, por lo que resulta adecuado que 


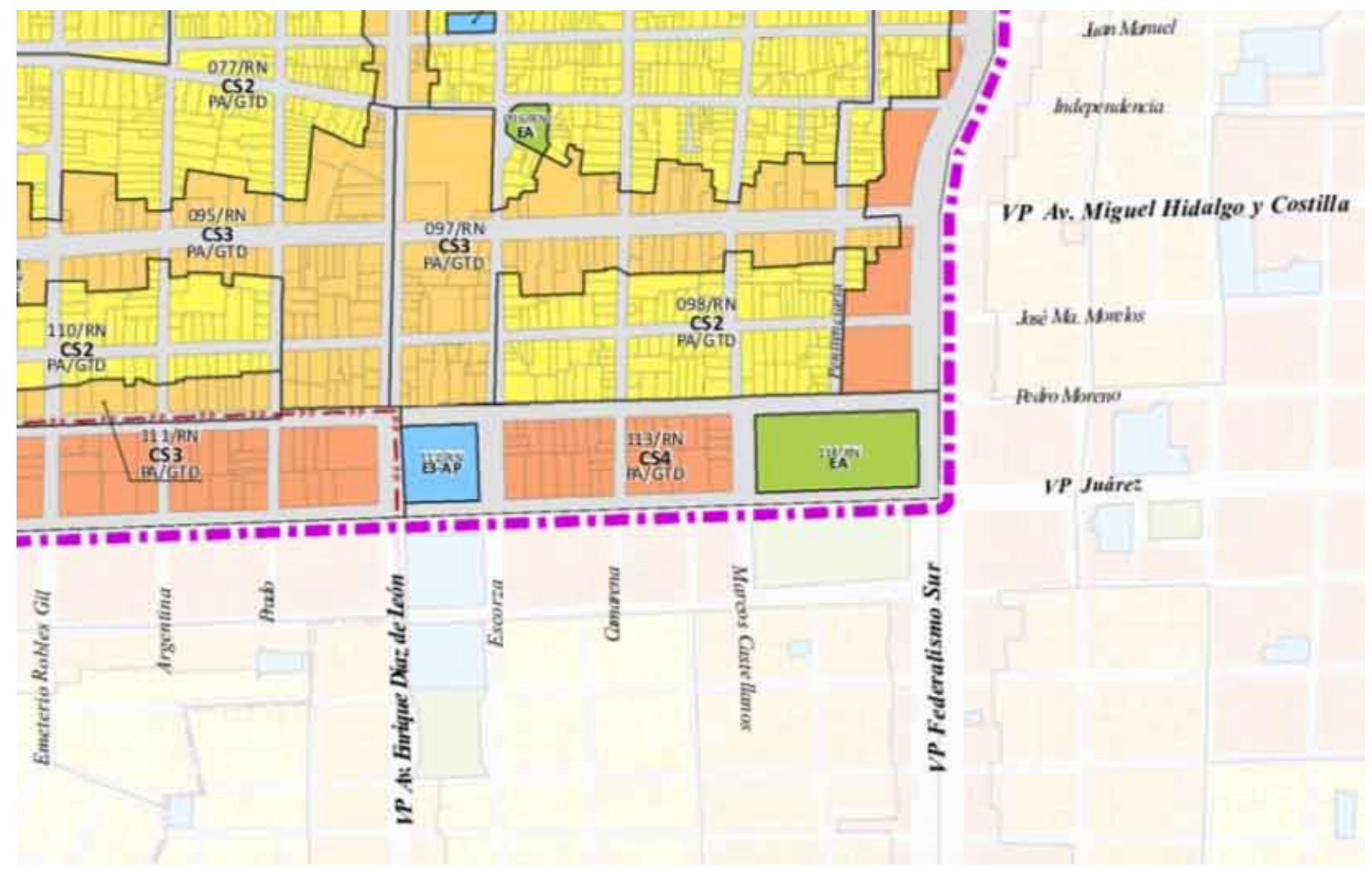

Ilustración 3. Plano de Zonificación Secundaria E3. Distrito Urbano 1 «Centro Metropolitano» y Subdistrito Urbano 04 «Santa Teresita». Plan Parcial de Desarrollo Urbano. Fuente: Gobierno de Guadalajara. 


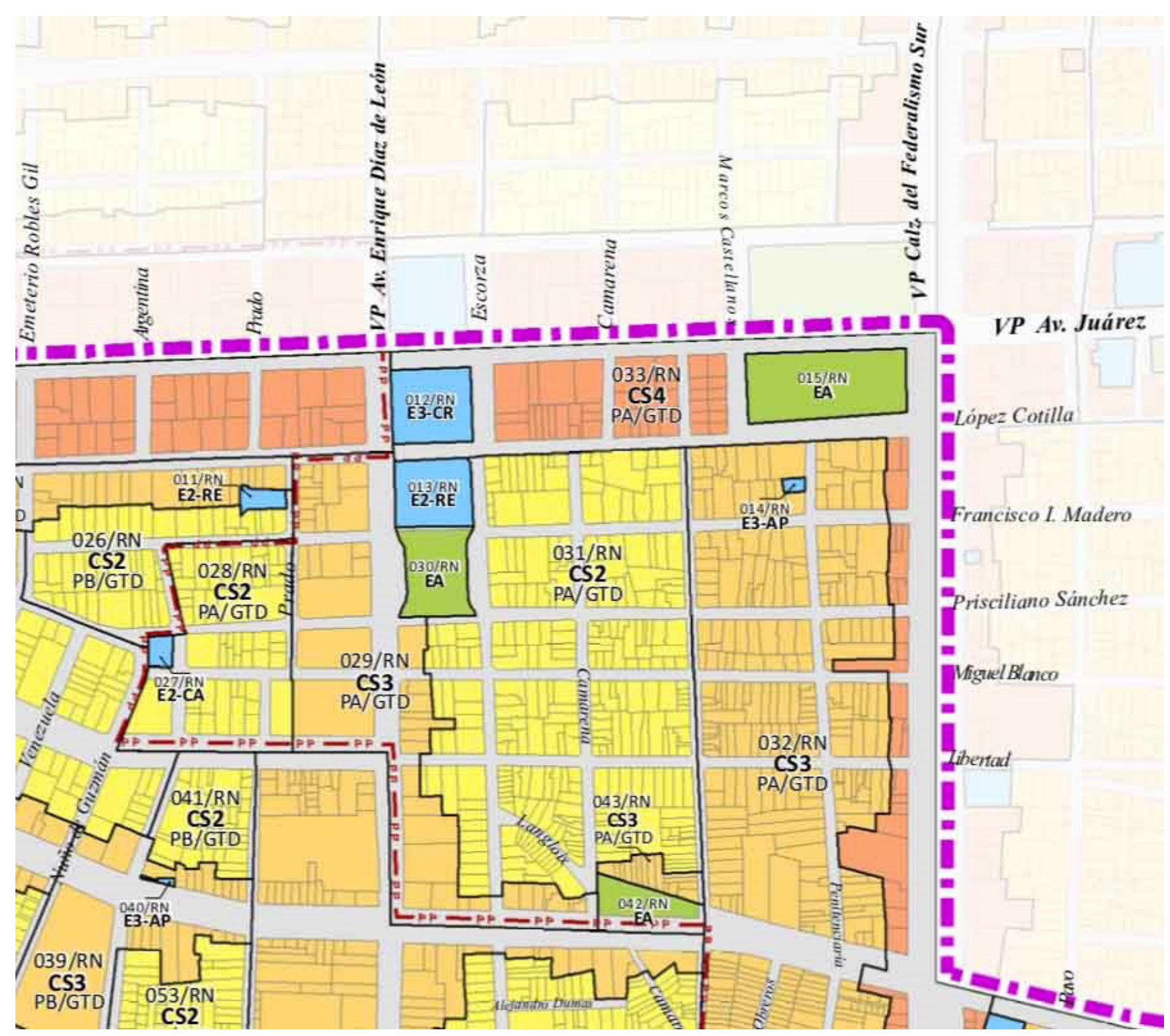

Ilustración 4. Plano de Zonificación Secundaria E3. Distrito Urbano 1 «Centro Metropolitano» y Subdistrito Urbano 07 «La Moderna». Plan Parcial de Desarrollo Urbano. Fuente: Gobierno de Guadalajara. 
se ubiquen sobre vialidades del sistema vial primario, como la avenida Juárez, con fácil accesibilidad hacia las salidas carreteras, como el Edificio Administrativo de la Universidad de Guadalajara y el $\mathrm{Mu}-$ seo de las Artes (MUSA), clasificados como equipamiento de Impacto Medio de Cultura (E3-C) según el Plan Parcial de Desarrollo Urbano. (Véase Ilustración 5).

El Museo de las Artes (MUsA) de la Universidad de Guadalajara, ubicado sobre la av. Juárez y la av. Enrique Díaz de León, exhibe de manera continua exposiciones de arte nacionales e internacionales, promovidas por la casa de estudios. Es necesario recordar que este mismo edificio es conocido como el Paraninfo de la Universidad de Guadalajara, que alberga un mural de José Clemente Orozco. ${ }^{7} \mathrm{Al}$ decidir acondicionar la parte de abajo del edificio como museo, la Universidad de Guadalajara, en 1994, consolidó esa zona de la ciudad como epicentro del arte en Guadalajara.

En esa dinámica, encontramos que, en la acera de enfrente, dentro del edificio administrativo de la Universidad de Guadalajara, se localiza el Cineforo Universidad, que es una importante sede de festivales nacionales e internacionales de cine, como el Festival Internacional de Cine de Guadalajara FICG, además de ser el espacio ideal para la proyección de cine documental y cinema de autor mexicano, latinoamericano, europeo y mundial.

Este encuentro entre lo intelectual y lo artístico refleja la esencia de la colonia Americana, la cual, junto con la colonia Francesa, conocida también como Lafayette (por el famoso francés que llegó a salvar a los independentistas estadounidenses de la derrota frente al imperio inglés a finales del siglo XVIII), representa la herencia europea en la perla tapatía.

A un costado del MUSA, tiene lugar los fines de semana el Corredor Cultural Expiatorio Rambla Cataluña, que realiza el comité de vecinos de la Colonia Americana desde abril del año 2012. Es un espacio cultural alternativo de conciencia social, donde se generan alternativas de diversión, esparcimiento y consumo a través diversas actividades culturales: música, teatro, danza, artesanía, cuenta cuentos, proyección de documentales, espacios de lectura, deporte y exposición y venta de productos orgánicos. También incluye cuestiones ecológicas y activación de economía local y solidaria.

Por otro lado, las zonas de espacios verdes abiertos y recreativos (EA), aun cuando forman parte del rubro del equipamiento, se clasifican de manera independiente de acuerdo con la importancia de las mismas para los centros de población. Con base en su área de influencia y actividades, pueden ser: centrales, como parques urbanos o jardines (por ejemplo, el parque Revolución o las plazas); distritales, que son espacios como la plaza del Agave; y vecinales, que son andadores peatonales, jardines vecinales, plazoletas y rinconadas como el parque Severo Díaz, ubicado en avenida De la Paz.

En ese sentido, la plaza del Agave es un espacio integrador de actividades comerciales, de convivencia y de esparcimiento, pero tam-

7 Autor del Hombre en llamas, su obra maestra, que se encuentra en el Instituto Cultural Cabañas, ubicado en el centro de esta misma ciudad. 
Ilustración 5. Museo de las Artes MUSA (Universidad de Guadalajara). Fuente: propiedad de la autora. bién es un espacio permanente de referencia para los estudiantes de las instituciones educativas próximas, aun cuando está dividido por avenidas de tráfico intenso que marcan límites urbanos muy fuertes.

El Templo Expiatorio constituye un hito del área de estudio. Para Lynch, los hitos o mojones son lugares específicos que pueden verse como límites o centros geográficos, estos indican un suceso, una dirección o un acontecimiento. También se pueden ver como un lugar de referencia, que puede ser un árbol, una escultura o un edificio. Son elementos invariables del espacio. Al tratarse de un análisis de índole cultural, la zona de estudio incluye gran parte de la colonia Americana, pero también algunas manzanas de dos barrios significativos: el barrio de los Artesanos y el barrio de Mexicaltzingo, que tienen una significación cultural importante y que en el pasado manifestaron prácticas y tradiciones culturales diferenciadas de las de la colonia Americana.

Al tratarse de un enfoque cultural, los límites de la zona no se adscriben a la zonificación urbana del Plan Parcial de Desarrollo Urbano del Ayuntamiento de Guadalajara, sino que permiten una flexibilidad en la delimitación de estudio. Estos límites de tránsito han generado dinámicas de convivencia diferenciadas a las que originalmente señala la división administrativa de la zona. Se observó que los límites de las avenidas Vallarta, Federalismo, La Paz y Enrique Díaz de León provocan que los usuarios de uno y otro extremo de las aceras se replieguen hacia su propio lado y eviten el lado opuesto.

Respecto a las funciones identitarias que para la colectividad cumplen ciertas condiciones, en este caso de culto, el Templo Expiatorio tiene también diversos matices, es un edificio ecléctico con una fuerte influencia neogótica y elementos de tradición arquitectónica italiana, que originalmente fue edificado para producir una identidad

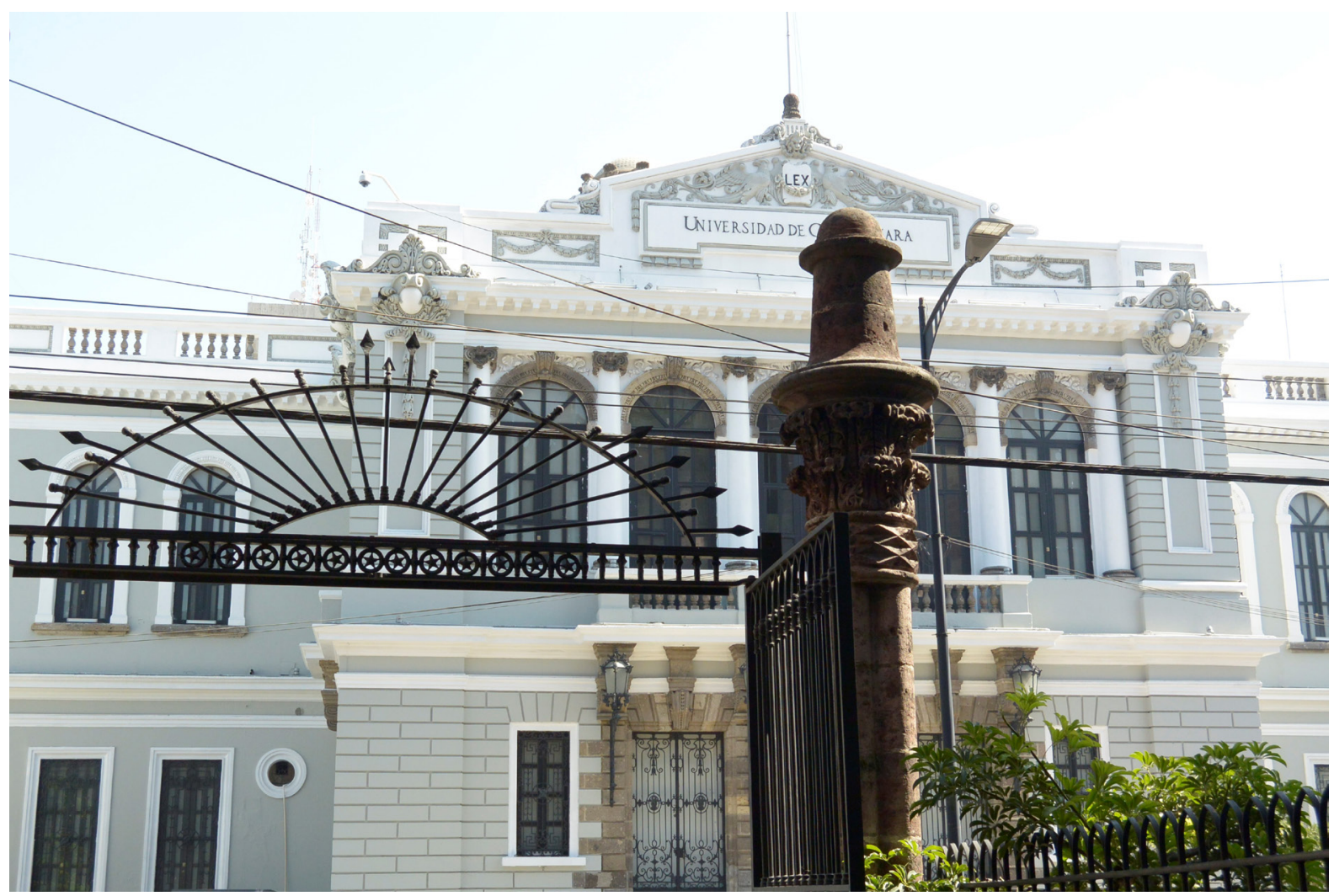


cultural de migrantes europeos en la capital de Guadalajara, función que quedó en el pasado, ya que estos elementos se han modificado en la memoria cultural de los habitantes de la ciudad. El recinto se ha convertido simplemente en un elemento de identidad religiosa que, aunque no es equiparable con otros edificios identitarios de Guadalajara, sí constituye un elemento de identidad regional, un hito. Pese a que ya no constituye un elemento de identidad dirigido a una comunidad de migrantes europeos, constituye un vestigio de la migración histórica que afectó a la ciudad. Actualmente tiene una función turística y patrimonial significativa.

Los hitos implican la selección de un elemento entre la multitud de posibilidades. Algunos de ellos están distantes y es característico que se les vea desde muchos ángulos y distancias, por arriba de las cúspides de elementos más pequeños y que se les utilice como referencias radiales. ${ }^{8}$

En esta lógica, existe un punto de coincidencia entre la visión antropológica del presente estudio y la zonificación del Plan Parcial de Desarrollo Urbano, ya que el uso de suelo del Templo Expiatorio está clasificado como Equipamiento Impacto Bajo Religioso (E2-RE). Este tipo de equipamiento forma núcleos de educación, cultura, salud, servicios y culto que sirven a amplias áreas del centro de población, generando centros distritales o subcentros urbanos, como es el caso de esta edificación religiosa.

El Templo Expiatorio del Santísimo Sacramento pertenece al estilo ecléctico neogótico, movimiento artístico surgido en el siglo XIX que imitaba la arquitectura gótica medieval. Desde un análisis estructuralista, el gótico se denomina estilema, que es un término para referirse a los rasgos característicos de un estilo determinado, al igual que a las marcas de estilo autoral, histórico y de género de una corriente o de una época.

El estilema está conformado por signos, sintagmas, elementos retóricos y otros componentes formales, así como por algunos elementos semánticos que permiten identificar estilísticamente una obra en relación con sus características objetivas, pero también en relación con su socialización, determinada por los valores sociales subyacentes.

Respecto a los comercios establecidos alrededor la plaza del Agave, son un indicador de los segmentos de la población que los visita. El hecho de que se trate de restaurantes y cafés propicia la convivencia en este espacio. Tal es el caso del restaurante El Fénix (que comenzó como una panadería y luego amplió su menú) y de la cafetería de La Flor de Córdoba. Se identifica también un Oxxo, franquicia que vino a sustituir a las tiendas de abarrotes barriales que antiguamente funcionaban en varias esquinas de la zona. (Véase la ilustración 6).

La avenida Enrique Díaz de León provocó un límite con respecto al mercado original del barrio, el Mercado Juárez, por lo que el espacio tradicional de comercio se segmentó por esta frontera imagina-

8 Lynch, La imagen de la ciudad, 61. 


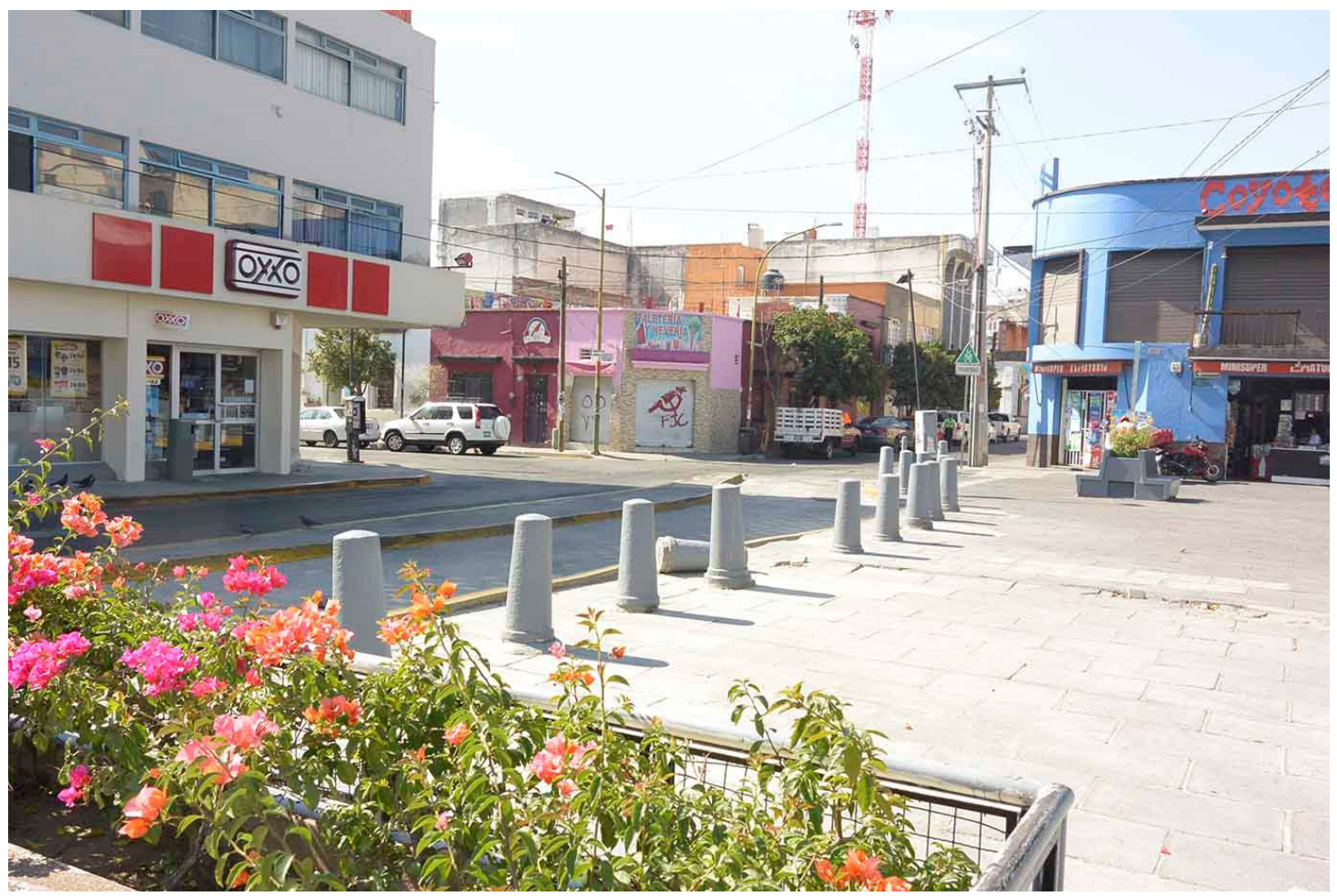

Ilustración 6. Vista de la plaza del Agave. Fuente: propiedad de la autora. ria, fue así que se desarrollaron nuevos comercios en la zona próxima al Templo Expiatorio, que todo espacio de convivencia requiere para satisfacer las necesidades básicas de sus visitantes.

El Instituto Cultural Mexicano y el Hospital Ramón Garibay, a pesar de estar separados por la misma avenida, se integran a la plaza del Agave, al menos en sus fachadas, y siguen cumpliendo con sus funciones fundamentales. Están clasificados como Servicios de Impacto Medio $\left(\mathrm{CS}_{3}\right)$ en una zona que sirve a la totalidad del centro de población. (Véase la ilustración 7).

Los espacios de integración del texto urbano están conformados por las manzanas que constituyen el cuerpo del barrio, así como los espacios de tránsito, es decir, calles y avenidas.

Las carreteras o sendas, son los conductos que sigue el observador normalmente, ocasionalmente o potencialmente. Están representadas por calles, senderos, líneas de tránsito, canales o vías férreas. Para muchas personas son éstos los elementos preponderantes en su imagen. Los sujetos observan la ciudad mientras utilizan estos espacios que organizan y conectan los demás elementos urbanos. ${ }^{9}$

Sobre la avenida Juárez existe una importante zona de comercios clasificada como Comercio y Servicios de Impacto Alto, a diferencia de las zonas sobre las avenidas Enrique Díaz de León y La Paz, que se presentan en Comercio y Servicios de Impacto Medio. Es una zona mixto-distrital al norte del área de estudio. Sobre la avenida Federalismo, se identifica una zona de Comercio y Servicios de Impacto Al-

9 Lynch, La imagen de la ciudad, 59. 
to, su zona de influencia es un distrito urbano, o el conjunto de varios barrios.

Generalmente estas zonas se constituyen alrededor de los subcentros urbanos o en corredores urbanos interzonales, siendo adecuadas para ubicar los usos de comercio y servicios de mayor impacto, así como las actividades de trabajo de baja incidencia en el medio ambiente.

En el cruce de la avenida Federalismo y la avenida Juárez se ubica el parque Revolución, proyecto histórico del arquitecto Luis Barragán. Este parque abarca cuatro manzanas, entre las actuales calles López Cotilla, Escobedo, de Jesús y Pedro Moreno. Está dividido en

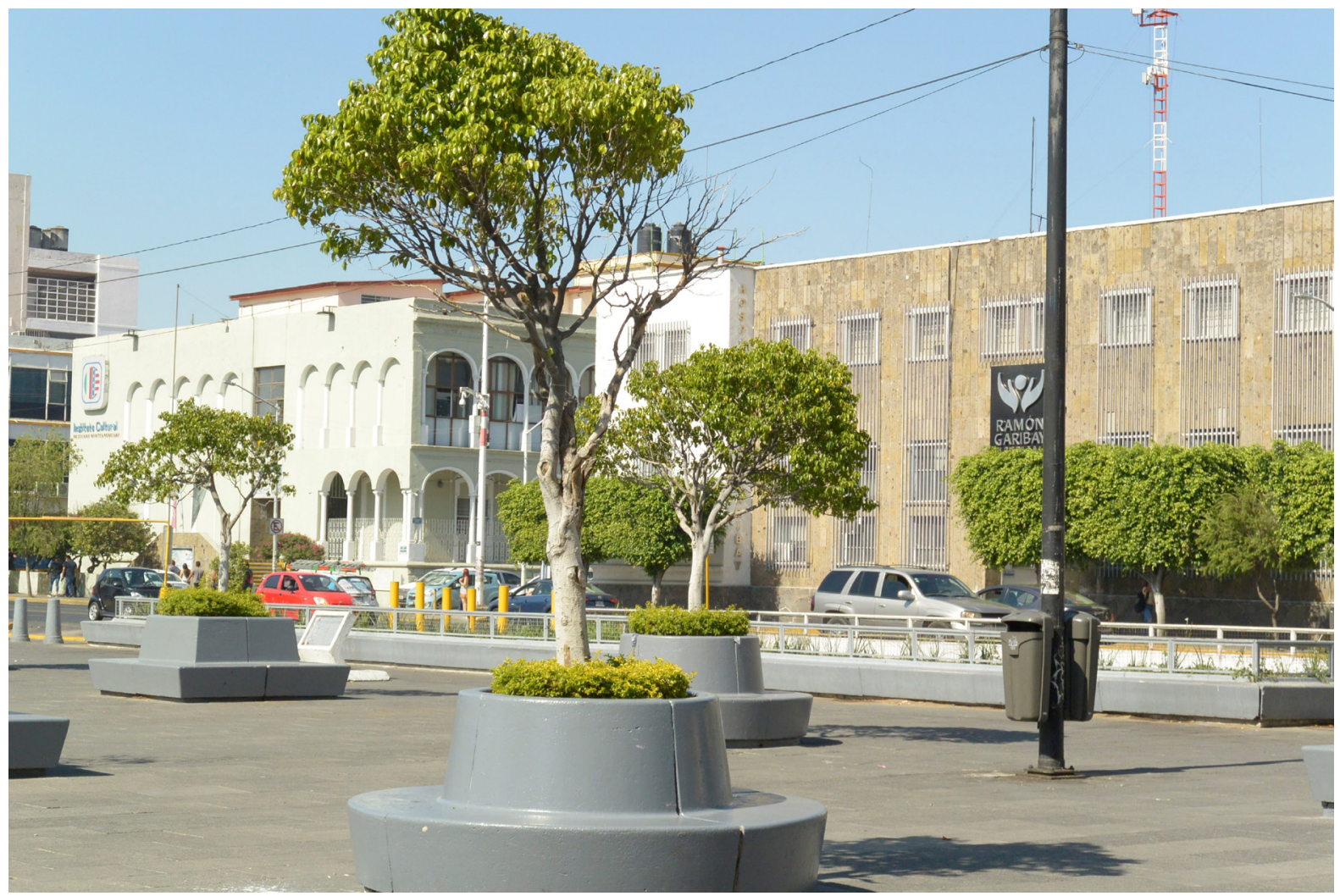

Ilustración 7. Instituto Cultural Mexicano y Hospital Ramón Garibay. Vista desde la plaza del Agave. Fuente: propiedad de la autora. dos partes por la avenida Juárez, que más adelante se convierte en Vallarta. La calle Penitenciaría y la avenida Juárez parten el parque en cuatro cuadrantes, de los cuales tres son prácticamente iguales y uno desigual, que es donde se encuentran el área de juegos y el área de servicios.

Este fue un proyecto previo a la etapa de madurez de Barragán (quien vivió después en la Ciudad de México), sin embargo, refleja el espíritu moderno de los años treinta. El parque fue considerado un proyecto contemporáneo en su momento, ya que también reflejó algunos conceptos de Le Corbusier y del funcionalismo europeo, desarrollados al inicio del siglo xx. (Véase la ilustración 8).

La paleta de colores está compuesta por rojo Pompeya y ocre, justamente los colores de la obra Les Colombiéres, del paisajista francés Ferdinand Bac, inspiración de Barragán. En un inicio, el parque tenía una glorieta central y un área de juegos infantiles (que nos recuerda los parques de los países europeos del siglo XIX), donde el arquitecto 
Ilustración 8. Parque de la Revolución. Fuente: propiedad de la autora. introdujo una atmósfera lúdica para niños, junto con piezas escultóricas en los elementos compositivos, dándole un aire de modernidad. Siguiendo también los patrones de la plaza tradicional y del parque victoriano, el proyecto tiene áreas para caminar, para deambular, para jugar, para sentarse y demás actividades similares.

El área original de juegos estaba perimetralmente cerrada por un muro con aberturas triangulares, a la manera de la vivienda vernácula jalisciense. Este muro tenía setos como limitante vegetal. Algunos elementos que destacan son el quiosco, el paraguas con banca y las luminarias de diseño modernista.

En sus inicios, este parque estaba rodeado por viviendas de clase alta. En los años setenta, el uso de estas edificaciones era mixto. Actualmente presenta un uso en su mayoría comercial. Este importante punto de encuentro constituye un área verde de recreación ciudadana céntrica y accesible, además, es terminal de varias rutas de transporte urbano. Sin embargo, ha atravesado por cambios drásticos de uso de suelo e imagen.

El diseño modernista del parque de la Revolución se contrapone en su ideología al diseño inicial del asentamiento que se analiza, que apuntaba hacia un modo de vida exterior a finales del siglo XIX, pues el concepto del arquitecto Barragán y sus proyectos instaban a enfocarse en la vida hacia el interior de la vivienda, lo cual era una manera de contraponerse a la ciudad caótica, dinámica y moderna.

En la actualidad, el parque de la Revolución marca una frontera en el área de estudio. Está ubicado justo arriba de la estación Juárez del Sistema de Tren Eléctrico Urbano (SITEUR), sitio donde la Secre-

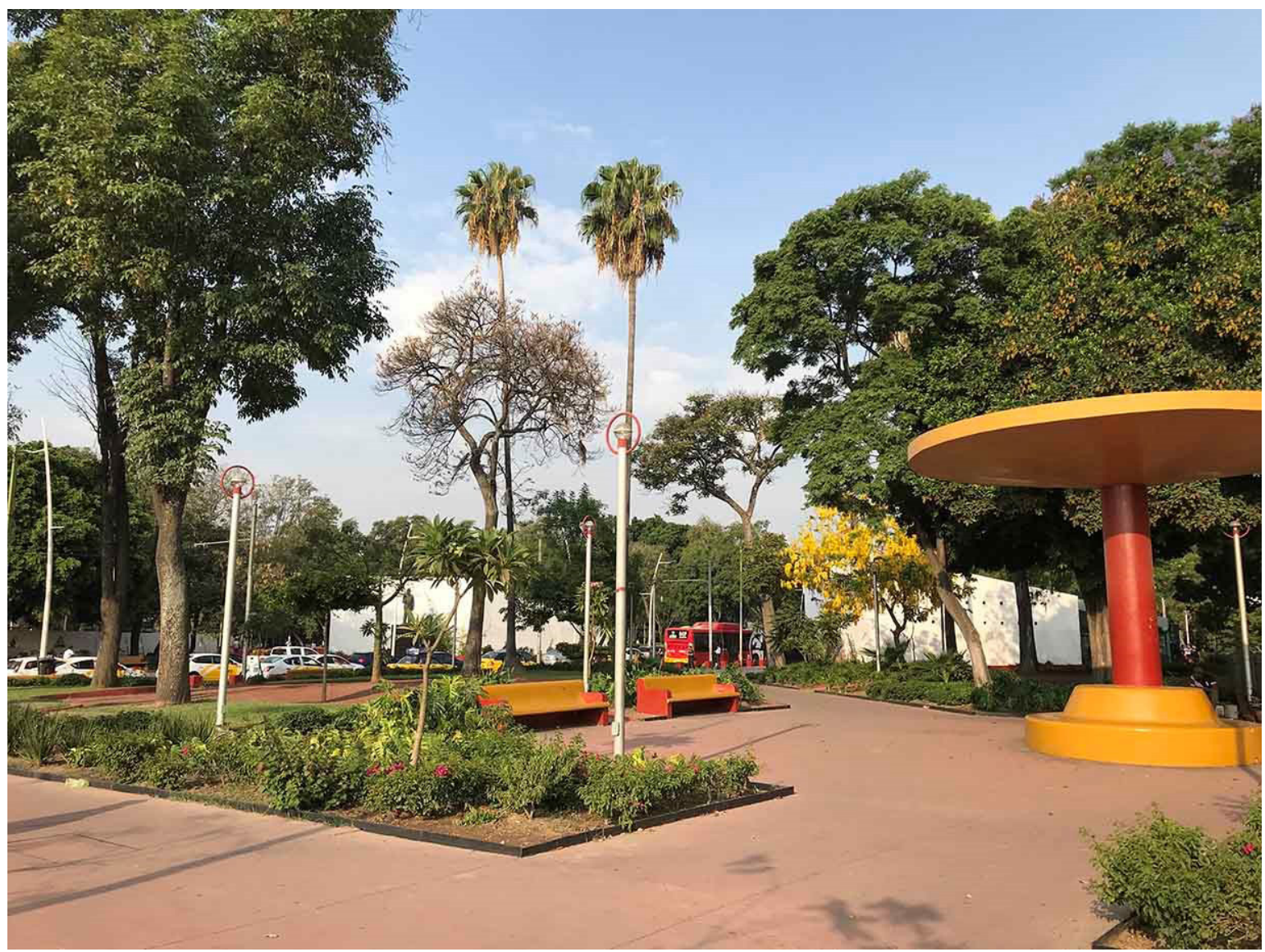


taría de Cultura de Guadalajara realiza continuamente exposiciones artísticas en la galería subterránea.

Los barrios son secciones de la ciudad concebidos de manera bidimensional, el observador entra en ellos en forma mental y le son reconocibles como si tuvieran un carácter que los identifica desde el interior pero también son visibles desde afuera como una referencia exterior. ${ }^{10}$

Según la clasificación de áreas, las manzanas al interior del área de estudio son áreas de Comercio y Servicios de Impacto Bajo, pertenecientes al Perímetro A, de la Zona de Protección del Centro Histórico (CS2 PA/GTD), así como Áreas Generadoras de Derechos de Desarrollo, con las instalaciones necesarias para la vida normal del centro de población, que cuentan con su incorporación municipal y pueden ser objeto de acciones de mejoramiento y de renovación urbana. Su uso es mixto-barrial.

Se consideran predominantes los usos habitacional unifamiliar, habitacional plurifamiliar horizontal y habitacional plurifamiliar vertical. Se consideran compatibles los usos: comercio vecinal, servicio vecinal, comercio barrial, servicio barrial, turístico hotelero, equipamiento vecinal, equipamiento barrial, espacios verdes, abiertos y recreativos vecinales, espacios verdes abiertos y recreativos barriales y manufacturas domiciliarias. En la actualidad se identificaron viviendas, galerías de arte, talleres de artistas, asociaciones civiles, cafés, bares, restaurantes y centros culturales.

Dentro del barrio fueron identificados centros culturales de iniciativa privada, como el Foro Periplo en la calle de Prisciliano Sánchez, lugar que promueve la movilidad y difusión de productos artísticos a nivel nacional e internacional, fomenta la creación artística y ofrece aulas para ensayo, práctica y reflexión de las manifestaciones escénicas. La casa teatro El Caminante es un recinto donde se presentan obras de teatro, conciertos, festivales y conferencias, ubicado en la calle de Marcos Castellanos 26. Se ubicaron dos colectivos: Colectivo 821, ubicado en la calle de Morelos, y Colectivos la percha, que son bazares de ropa, libros, discos, revistas, antigüedades y artículos de belleza.

En estas zonas la habitación es predominante, pero compatible con otros usos comerciales y de servicios estrictamente barriales. En ellas, el uso habitacional, según el Plan Parcial, no podrá ser menor de 75 por ciento de la zona. Generalmente se constituyen alrededor de los centros vecinales o centros de barrio, o en corredores internos del barrio.

Cada capítulo de la historia dejó registro en el paisaje urbano del barrio. Tal es el caso de la demolición de la Escuela de Música de la Universidad de Guadalajara (que estaba ubicada en el cruce de la Av. Juárez y Enrique Díaz de León), edificio neoclásico del arquitecto Alfredo Navarro Branca que, pese a haber sido una edificación protegida por el Instituto Nacional de Bellas Artes del Ayuntamiento de

10 Lynch, La imagen de la ciudad, 60. 


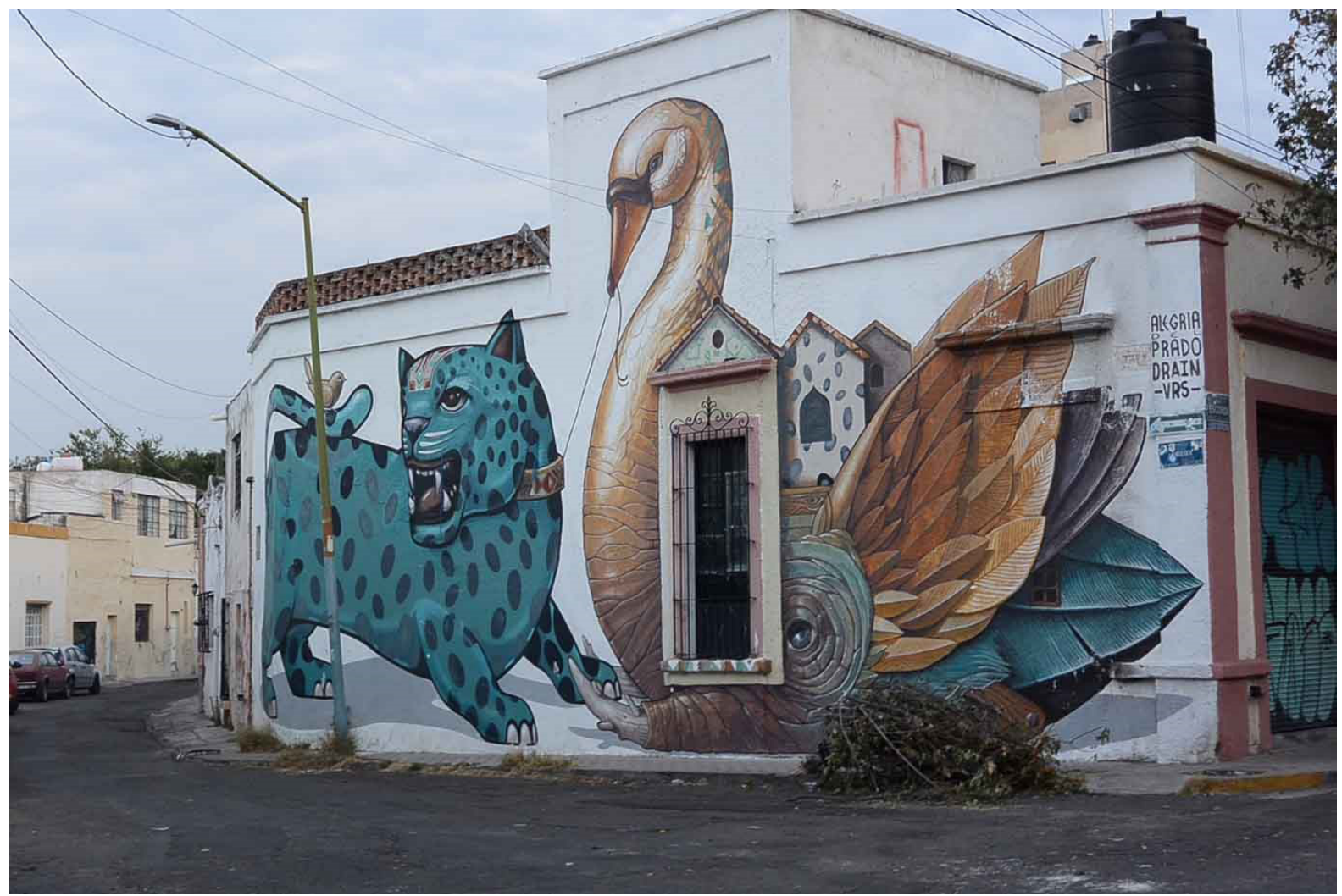

Ilustración 9. Grafiti en la esquina de la calle Escorza y Langloix. Fuente: propiedad de la autora. la ciudad, fue demolido en marzo de 1980. En su lugar actualmente se encuentra el Edificio Cultural y Administrativo de la casa de estudios, hecho que disolvió la identidad de la ciudad como centro de cultura musical.

Desde el neogótico del Templo Expiatorio, el estilo renacentista francés del Museo de las Artes, antes Rectoría General de la Universidad de Guadalajara, y la corriente modernista del parque Rojo, así como la mezcla de art nouveau, ecléctico francés, neocolonial, regionalismo y revivals en las viviendas que conforman este collage arquitectónico, es posible realizar una lectura del paisaje urbano que da cuenta de los contextos históricos, económicos y sociales de la ciudad.

\section{Conclusiones}

Uno de los retos de la antropología urbana es superar el carácter descriptivo de datos y explicar a través del análisis de procesos históricos las dinámicas que estructuran el desarrollo urbano. En este sentido, partiendo de información documental y etnográfica del barrio se ha logrado establecer un diálogo entre los modelos teóricos elegidos y el análisis cultural de la zona.

Este análisis se inscribe en una tradición de antropología interpretativa, desde una corriente estructuralista que parte de las semejanzas de las culturas antes que de las diferencias, y pretende redefinir tanto el objeto de estudio como el método, en oposición al positivismo 
dominante, otorgando una importancia fundamental a los símbolos y significados culturalmente compartidos.

En la antropología, la verificación se realiza a través del trabajo de campo y la observación participante, que conduce a conclusiones sistemáticas y argumentadas. Esta metodología cualitativa remite a una elaboración teórica de la cultura humana como objeto de estudio que la vincula con otras disciplinas de las ciencias sociales y humanidades, tales como la arquitectura, la filosofía, la geografía, el urbanismo y la historia.

La poética del espacio constituye la materia de análisis de este "conjunto estructural simbólico (CES)». ${ }^{11}$ A través de componentes verticales y horizontales el medio ambiente fue estructurado de manera pasiva y activa en los distintos niveles de uso, desde las viviendas, las manzanas, las calles y el barrio como unidades significantes. La integración de los barrios como unidades estructurantes es lo que conformó la ciudad de Guadalajara.

La transformación del paisaje urbano es el texto que se pretende leer, entender y apropiar no solo desde una mirada exterior sino de adentro hacia fuera enfatizando que el sujeto y las identidades son posiciones determinadas socialmente e ideológicamente estructuradas.

\section{Bibliografía}

Amerlinck, Mari-Jose. Hacia una antropología arquitectónica. $1^{\mathrm{a}}$ edición. Guadalajara, Jalisco: Universidad de Guadalajara, 1995.

Bailly, Antoine. La percepción del espacio urbano. Madrid: Instituto de Estudios de Administración Local, 1979.

Bolaños Palacios, Javier, Pachajoa, José Tomas, y Aguilera Martínez, Fabián. «Concepto de edilicia.» Revista de Arquitectura (Universidad Católica de Colombia), núm. 6 (2004): 38-39.

Broadbent, Geoffrey, Bunt, Richard, y Jencks, Charles. El lenguaje de la arquitectura: un análisis semiótico. México: Limusa, 1991.

Eco, Umberto. La estructura ausente. Introducción a la semiótica. España: Lumen, 1986.

Egenter, Nold. «2. Antropología arquitectónica: un nuevo enfoque antropológico». En Hacia una antropología arquitectónica, edita-

11 Egenter, «Antropología arquitectónica: un nuevo enfoque antropológico», 69. 
do por Mari-Jose Amerlinck. Guadalajara, Jalisco: Universidad de Guadalajara, 1995.

Gobierno de Guadalajara. Página Oficial del Gobierno de Guadalajara 2017. Recuperado de <https://guadalajara.gob.mx/planes-parciales/>. (último acceso: 10 de octubre de 2019).

Lynch, Kevin. La imagen de la ciudad. $3^{\text {a }}$ edición. Barcelona: Gustavo Gili, 2015.

Sanders Peirce, Charles. La ciencia de la semiótica. Buenos Aires: Ediciones Nueva Visión, 1986.

Secretaría de Desarrollo Social (sedesol). «Tomo I. Educación y Cultura.» En Sistema Normativo de Equipamiento Urbano, editado por Gobierno de México, 2018.

Smithson, Alison. Team 10 Primer. Londres: Studio Vista, 1968.

Todorov, Tzvetan. Poética estructuralista. $1^{\text {a }}$ edición. Barcelona: Losada, 2004. 